\title{
In silico design of a Zika virus non-structural protein 5 aiming vaccine protection against zika and dengue in different human populations
}

\author{
Lorrany dos Santos Franco, Paloma Oliveira Vidal and Jaime Henrique Amorim * (1)
}

\begin{abstract}
Background: The arboviruses Zika virus (ZIKV) and Dengue virus (DENV) have important epidemiological impact in Brazil and other tropical regions of the world. Recently, it was shown that previous humoral immunity to DENV enhances ZIKV replication in vitro, which may lead to more severe forms of the disease. Thus, traditional approaches of vaccine development aiming to control viral infection through neutralizing antibodies may induce cross-reactive enhancing antibodies. In contrast, cellular immune response was shown to be capable of controlling DENV infection independently of antibodies. The aim of the present study was to design a flavivirus NS5 protein capable of inducing a cellular immune response against DENV and ZIKV.
\end{abstract}

Methods: A consensus sequence of ZIKV NS5 protein was designed among isolates from various continents. Epitopes were predicted for the most prevalent alleles of class I and II HLA in the Brazilian population. Then, this epitopes were analyzed with regard to their conservation, population coverage and distribution along the whole antigen.

Results: Nineteen epitopes predicted to be more reactive (percentile rank $<1$ ) and 100\% conserved among ZIKV and DENV serotypes were selected. The distribution of such epitopes along the protein was shown on a three-dimensional model and population coverage was calculated for different regions of the world. The designed protein was predicted to be stable and the distribution of selected epitopes was shown to be homogeneous along domains. The population coverage of selected epitopes was higher than $50 \%$ for most of tropical areas of the world.

Conclusion: Such results indicate that the proposed antigen has the potential to induce protective cellular immune response to ZIKV and DENV in different human populations of the world.

Keywords: Zika virus, Dengue virus, Vaccine, Epitopes, Bioinformatics

\section{Background}

The Flavivirus genus of the Flaviviridae family consists of more than 70 viruses, many of which are arthropod-borne viruses, i.e. arboviruses [1]. It is estimated that more than 390 million people are infected annually by any of the four serotypes of Dengue virus (DENV1-4) [2], the most prevalent of the emerging arboviruses. Recently, Zika virus (ZIKV), an emergent pathogen previously associated with mild infections, started to be associated with microcephaly

\footnotetext{
* Correspondence: jaime.amorim@ufob.edu.br

Laboratório de Microbiologia, Centro das Ciências Biológicas e da Saúde, Universidade Federal do Oeste da Bahia, Rua Bertioga, 892, Morada Nobre II, Barreiras, Bahia CEP 47810-059, Brazil
}

in babies and Guillain-Barré syndrome in the Americas [3-5]. Together, these two Flavivirus consist a world public health concern for which there is not specific treatment. For now, controlling arthropod vectors is the most effective and available method to prevent DENV and ZIKV burden. However, effective vaccines are needed in order to complement mosquito control programs, once that eradication of vectors is challenging and time consuming.

The only anti-DENV vaccine candidate approved for use in humans is based on a chimera between DENV and Yellow fever virus (YFV) [6-10]. It consists of chimeric viruses for each of DENV serotypes in which 
genomic sequences coding for YFV envelope proteins were replaced by those of DENV. Thus, specific immunity against DENV is concentrated on antigens that mainly induce generation of antibodies. Unfortunately, especially for DENV2, the vaccine formulation based on those chimeric viruses did not achieve the expected protective efficacy in phase III clinical trials in different regions of the world. In addition, it was reported to present higher incidence of hospitalization for dengue in year 3 after vaccination among children younger than 9 years of age [10]. Some severe forms of dengue are mediated by a phenomenon called antibody-dependent enhancement, in which immunoglobulins produced in response to a previous DENV serotype cross-react with viral particles of a second serotype and mediate enhanced infection of Fc- $\gamma$ receptor bearing cells. Such enhanced infection leads to higher viral loads and severe disease. Relevantly, such phenomenon was reported between DENV and ZIKV [11-14]. Thus, the risk of inducing antibody-mediated enhancement of infection between these two major arboviruses will depend on the profile of immune response induced.

In contrast to envelope proteins, which are structural proteins, non-structural proteins are not present in viral particles and are not able to contribute directly to antibody-dependent enhancement. In addition, they were shown to be major targets for $\mathrm{CD}^{+}$and $\mathrm{CD} 8^{+} \mathrm{T}$ lymphocytes involved with control of DENV spread and intracellular replication [15-17]. Also, $\mathrm{CD}^{+} \mathrm{T}$ lymphocytes with multifunctional cytokine secretion patterns were found in volunteers immunized with a live attenuated tetravalent dengue vaccine [18]. Such lymphocytes target highly conserved epitopes located on non-structural proteins and this immunological pattern fits with those found after natural infections in which control of disease was observed [18]. Moreover, our last reports showed that protection against DENV is achieved independently of humoral immunity [19]. $\mathrm{CD}^{+}$and $\mathrm{CD}^{+}{ }^{+} \mathrm{T}$ lymphocytes targeting non-structural proteins, mainly NS5, were shown to be essential for protection capacity. When a recombinant purified form of NS5 protein was used as a vaccine antigen, we achieved $70 \%$ of protection working on a mouse model [20].

The NS5 protein is a well-conserved antigen between DENV and ZIKV. It is the major DENV target for cellular immune response. In this study we aimed to design a vaccine antigen capable of inducing cross-protective cellular immunity against DENV and ZIKV. Here, we predicted NS5 common epitopes for DENV and ZIKV to be presented by HLA (human leucocyte antigen) encoded by alleles of different populations of the world. The designed antigen was shown to present a homogeneous distribution of epitopes and its predicted 3D model was shown to fit with the structure of a ZIKV native NS5. The population coverage of the antigen was higher than $50 \%$ for most of tropical regions of the world. Thus, it is possible to predict vaccine efficacy by region. In summary, we present a designed antigen which may be a valuable alternative in order to control the burden of DENV and ZIKV.

\section{Methods}

NS5 sequences database building

One database was built with NS5 protein amino acid sequences from ZIKV and DENV isolated in different continents of the world. Sequences in FASTA format were retrieved from the National Center for Biotechnology Information (NCBI) protein database (http:// www.ncbi.nlm.nih.gov/protein/). Criteria for selecting sequences were: i) complete annotation of the NS5 protein and ii) absence of undefined amino acid in the sequence. The database consisted of 153 NS5 protein amino acid sequences from the four serotypes of DENV and of 41 NS5 protein amino acid sequences ZIKV isolates. A sequence of NS5 protein from Spondwedi virus (accession number: ABI54480.1) was included to serve as a control. Accession numbers of DENV and ZIKV NS5 sequences are shown in Additional file 1: Tables S1 to S5.

\section{Multiple alignment, consensus sequence design and phylogeny of the ZIKV sequences}

Evolutionary analyses were conducted in MEGA7. Multiple Alignments of DENV and ZIKV sequences were carried out using the ClustalW method [21]. A NS5 consensus sequence among ZIKV isolates was designed based on results obtained from multiple alignment of ZIKV NS5 proteins, using MegAlign program from Lasergene. The evolutionary history of DENV and ZIKV NS5 proteins was inferred using the Neighbor-Joining method. Spondwedi virus was used as a control. A bootstrap test of 1000 replicates was applied. The evolutionary distances were computed using the Poisson correction method. The analysis involved 195 amino acid sequences.

\section{Survey of the most frequent HLA alleles in the Brazilian population}

The allelic frequency of both, class I and class II HLA, in the Brazilian population, was retrieved from NCBI database (https://www.ncbi.nlm.nih.gov/projects/gv/mhc/ihwg.cgi). Alleles frequent in at least $3 \%$ of the Brazilian population were selected.

\section{Epitope prediction analysis}

Epitopes within the NS5 protein consensus were predicted using the IEDB (Immune epitope database) analysis resource (http://tools.immuneepitope.org/mhci/), IEBD recommended method. NetMHCpan method was also used when prediction was not possible by using IEBD 
recommended method. The epitopes from the NS5 consensus were ranked by their percentile rank value and those with a percentile rank $\leq 1$ were selected. Epitopes with 8, 9, 10 and 11 amino acids in length were considered for Class I HLA and 15 amino acids for Class II HLA. A file containing the best ranked epitopes was created.

\section{Analysis of conservancy of predicted epitopes}

The IEDB conservancy analysis tool (http://tools.iedb.org/ conservancy) was used to determine the conservancy of the predicted epitopes among ZIKV lineages and DENV serotypes. Only predicted epitopes with a percentile rank $\leq 1$ were used in this analysis. Only epitopes $100 \%$ conserved among all sequences of DENV and ZIKV were selected.

\section{Population coverage analyses}

Epitopes previously selected were used to determine the population coverage by the IEDB population coverage calculation tool (http://tools.immuneepitope.org/tools/ population/iedb_input). All of the selected epitopes were analyzed for similarity with human proteome using BLAST program (http://www.ncbi.nlm.nih.gov/BLAST/) to verify if they would not trigger autoimmunity.

\section{Structural biology analysis}

The ZIKV NS5 consensus amino acid sequence was used to compute a 3D model using the I-TASSER modeling method. The best computed model was subjected to TM-align structural alignment program to match the first I-TASSER model to all structures in the PDB library (Protein Data Bank - http://www.rcsb.org). The top 1 model was also used to determine epitope distribution in protein domains. Epitopes were marked in the NS5 protein 3D model using the PyMOL program aiming to access their distribution.

\section{Results \\ ZIKV NS5 phylogeny}

In order to verify the origin of ZIKV isolates from Brazil and the Americas we carried out a phylogenetic analysis with NS5 amino acid sequences from ZIKV from throughout the world. We also included NS5 sequences from DENV serotypes from different genotypes isolated in different continents in order to verify similarities among ZIKV and DENV proteins. A comprehensive phylogenetic analysis of NS5 amino acid sequences based on world representative strains of ZIKV and DENV is shown in Fig. 1. NS5 sequences from Brazilian and American isolates grouped with those from the Asian lineage of ZIKV. None of the NS5 sequences from American isolates grouped with those from the African lineage of ZIKV. This result indicates that Brazilian and American ZIKV isolates analyzed in this study belong to the Asian lineage.
In addition, the phylogeny indicates that both, DENV and ZIKV NS5 proteins diverged before their separation into two different viral species. In other words: proteins diverge since the root of the phylogenetic tree.

\section{Consensus sequence design}

Although it has been shown that Brazilian and American ZIKV isolates belong to the Asian lineage, all the sequences contained in ZIKV database were used in the design of the consensus. It means that African sequences were also used. The option for including them was based on the aim of preventing disease with imported viruses from African lineage. Attempts to design a consensus NS5 protein between ZIKV and DENV resulted in a non-stable 3D model (data not shown). The ZIKV NS5 consensus is shown in Fig. 2.

\section{The most frequent alleles in Brazilian population were selected}

The survey of HLA frequencies in Brazilian population demonstrated that the most frequent are those in Table 1. Eleven alleles were selected for HLA-A: A*01:01, A*02:01, $A^{*} 02: 02, \quad A * 02: 24, A * 03: 01, A^{*} 11: 01, A * 24: 02, A * 29: 02$, $A * 31: 01, A^{*} 33: 03$ e $A * 68: 02$. Seven alleles were selected for HLA-B: B*07:02, B*08:01, B*08:05, B*18:01, B*35:01, $B * 44: 03, B * 51: 01$. Eleven alleles were selected for HLA-C: C*02:02, C*03:03, C*03:04, C*04:01, C*05:01, C*07:01, C*07:02, C*08:02, C*12:03, C"15:02, C*17:01 and thirteen for HLA-DRB1 (DRB1*01:01, DRB1*01:02, DRB1*03:01, DRB1*04:01， DRB1*07:01， DRB1*08:04， DRB1*11:01, DRB1*11:04, DRB1*13:01, DRB1*13:02, DRB1*14:01, DRB1*15:01, DRB1*15:03).

\section{Epitope prediction and conservation analyses}

Epitopes with higher binding affinity to the HLA molecules selected before were identified. Then, they were analyzed with regard to their conservation in all the ZIKV NS5 sequences contained in the database previously prepared. Epitopes with percentile rank $\leq 1$ (high binding affinity) and 100\% conserved among ZIKV and DENV NS5 sequences were selected. We selected 19 epitopes (Table 2) $100 \%$ conserved among all DENV and ZIKV NS5 amino acid sequences analyzed in this study. This result indicates that NS5 protein concentrates a relevant number of epitopes highly conserved among ZIKV lineages and DENV serotypes. In addition, conservation analyses also showed that 624 epitopes are $100 \%$ conserved among ZIKV lineages, 85 epitopes are 100\% conserved among ZIKV and DEN1, 102 epitopes are 100\% conserved among ZIKV and DEN2, 61 epitopes are 100\% conserved among ZIKV and DEN3 and 126 epitopes are 100\% conserved among ZIKV and DEN4. This result indicates that the consensus ZIKV NS5 protein share different numbers of epitopes with each of your homologous proteins. 


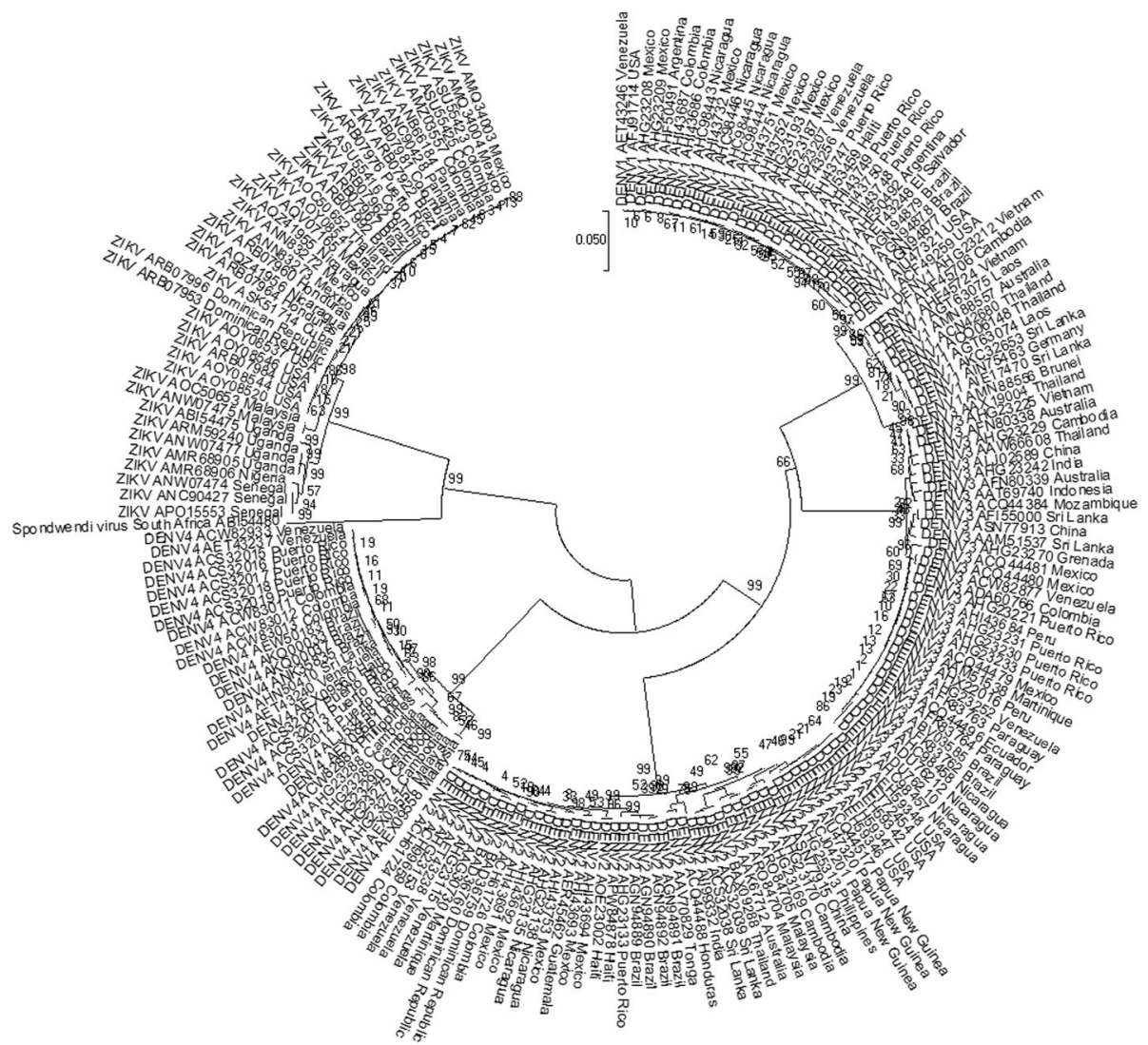

Fig. 1 Evolutionary history of selected amino acid sequences of ZIKV and DENV NS5 proteins. The tree shows that NS5 sequences from Brazilian ZIKV isolates grouped with those from Asian ZIKV lineage. The evolutionary history was inferred by using the Maximum Likelihood method based on the JTT matrix-based model [1]. The percentage of trees in which the associated taxa clustered together is shown next to the branches. Initial tree(s) for the heuristic search were obtained automatically by applying Neighbor-Join and BioNJ algorithms to a matrix of pairwise distances estimated using a JTT model, and then selecting the topology with superior log likelihood value. The tree is drawn to scale, with branch lengths measured in the number of substitutions per site. The analysis involved 195 amino acid sequences. All positions containing gaps and missing data were eliminated. There were a total of 896 positions in the final dataset. Evolutionary analyses were conducted in MEGA7

\section{Population coverage of epitopes predicted in ZIKV NS5} consensus vary in different human populations of the planet As shown in conservancy analyses, we selected nineteen epitopes with $100 \%$ identity among all the ZIKV and DENV NS5 sequences from databases. Such epitopes were shown to present a relevant population coverage with regard to the most prevalent HLA alleles in the Brazilian population, ranging from $3.9 \%$ to $33.73 \%$ (Table 2 ). Together, they presented accumulated population coverages of $74.07 \%$ and $68.70 \%$ for Brazilian and United States

\begin{abstract}
ZIKV NS5 protein consensus sequence
GGGTGETLGEKWKARLNQMSALEFYSYKKSGITEVCREEARRALKDGVATGGHAVSRGSAKLRWLVERGYLQPYGKVID LGCGRGGWSYYAATIRKVQEVKGYTKGGPGHEEPVLVQSYGWNIVRLKSGVDVFHMAAEPCDTLLCDIGESSSSPEVEEA RTLRVL SMVGDWLEKRPGAFCIKVLCPYTSTMMETLERLQRRYGGGLVRVPLSRNSTHEMYWVSGAKSNTIKSVSTTSOT LLGRMDGPRRPVKYEEDVNL GSGTRAVVSCAEAPNMKIIGNRIERIRSEHAETWFFDENHPYRTWAYHGSYEAPTQG SASSLINGVVRLLSKPWDVVTGVTGIAMTDTTPYGQQRVFKEKVDTRVPDPQEGTRQVMSMVSSWLWKELGKHK RPRVCTKEEFINKVRSNAALGAIFEEEKEWKTAVEAVNDPRFWALVDKEREHHLRGECQSCVYNMMGKREKKQ GEFGKAKGSRAIWYMWLGARFLEFEALGFLNEDHWMGRENSGGGVEGLGLORLGYVLEEMSRIPGGRMYADD TAGWDTRISRFDLENEALITNQMEKGHRALALAIIKYTYQNKVVKVLRPAEKGKTVMDIISRQDQRGSGQVVTYAL NTFTNLVVQLIRNMEAEEVLEMQDLWLLRRSEKVTNWLQSNGWDRLKRMAVSGDDCVVKPIDDRFAHALRFLN DMGKVRKDTQEWKPSTGWDNWEEVPFCSHHFNKLHLKDGRSIVVPCRHQDELIGRARVSPGAGWSIRETACLAK SYAQMWQLLYFHRRDLRLMANAICSSVPVDWVPTGRTTWSIHGKGEWMTTEDMLVVWNRVWIEENDHMEDKT PVTKWTDIPYLGKREDLWCGSLIGHRPRTTWAENIKNTVNMVRRIIGDEEKYMDYLSTQVRYLGEEGSTPGVL
\end{abstract}

Fig. 2 Consensus sequence of the ZIKV NS5 protein. Amino acid sequences from ZIKV NS5 database were used to construct a consensus sequence. The methyltransferase MTase domain is shown in gray, the linker region between MTase and RdRp domains is shown in black and underlined and the RdRp domain is shown black and bold 
Table 1 Frequency of class I and class II HLA alelles ( $\geq 3 \%$ ) in the Brazilian population

\begin{tabular}{|c|c|c|c|}
\hline Class & Loci & Alelle & Frequence \\
\hline \multirow[t]{29}{*}{ Class I } & \multirow[t]{11}{*}{ HLA-A } & $A^{*} 01: 01$ & 0.091 \\
\hline & & $A^{*} 02: 01$ & 0.192 \\
\hline & & $A^{*} 02: 02$ & 0.040 \\
\hline & & $A^{*} 02: 24$ & 0.040 \\
\hline & & $A^{*} 03: 01$ & 0.051 \\
\hline & & $A^{*} 11: 01$ & 0.061 \\
\hline & & $A^{*} 24: 02$ & 0.081 \\
\hline & & $A^{*} 29: 02$ & 0.030 \\
\hline & & $A^{*} 31: 01$ & 0.040 \\
\hline & & $A^{*} 33: 03$ & 0.030 \\
\hline & & $A^{*} 68: 02$ & 0.035 \\
\hline & \multirow[t]{7}{*}{ HLA-B } & $B^{* 07: 02}$ & 0.101 \\
\hline & & $B^{*} 08: 01$ & 0.043 \\
\hline & & $B^{*} 08: 05$ & 0.036 \\
\hline & & $B^{*} 18: 01$ & 0.051 \\
\hline & & $B^{*} 35: 01$ & 0.051 \\
\hline & & $B^{*} 44: 03$ & 0.035 \\
\hline & & $B * 51: 01$ & 0.051 \\
\hline & \multirow[t]{11}{*}{ HLA-C } & $C^{*} 02: 02$ & 0.033 \\
\hline & & $C^{*} 03: 03$ & 0.048 \\
\hline & & $C^{*} 03: 04$ & 0.048 \\
\hline & & $C^{*} 04: 01$ & 0.138 \\
\hline & & $C^{*} 05: 01$ & 0.062 \\
\hline & & $C^{*} 07: 01$ & 0.157 \\
\hline & & $C^{*} 07: 02$ & 0.081 \\
\hline & & $C^{*} 08: 02$ & 0.076 \\
\hline & & $C^{*} 12: 03$ & 0.038 \\
\hline & & $C^{*} 15: 02$ & 0.033 \\
\hline & & $C^{*} 17: 01$ & 0.033 \\
\hline \multirow[t]{13}{*}{ Class II } & \multirow[t]{13}{*}{ HLA-DRB1 } & DRB $1 * 01: 01$ & 0.055 \\
\hline & & DRB $1 * 01: 02$ & 0.035 \\
\hline & & DRB $1 * 03: 01$ & 0.121 \\
\hline & & DRB $1 * 04: 01$ & 0.035 \\
\hline & & DRB $1 * 07: 01$ & 0.075 \\
\hline & & DRB $1 * 08: 04$ & 0.030 \\
\hline & & DRB ${ }^{*} 11: 01$ & 0.060 \\
\hline & & DRB $1 * 11: 04$ & 0.035 \\
\hline & & DRB ${ }^{*} 13: 01$ & 0.070 \\
\hline & & DRB $1 * 13: 02$ & 0.050 \\
\hline & & DRB1*14:01 & 0.035 \\
\hline & & DRB1*15:01 & 0.050 \\
\hline & & DRB $1 * 15: 03$ & 0.045 \\
\hline
\end{tabular}

populations, respectively. In the Brazilian population, coverages found by ethnicity were $66.07 \%$ for Amerindian, $46.71 \%$ for Caucasoid, and $75.63 \%$ for Mixed. In the population of United States, coverages found by ethnicity were $50.64 \%$ for Amerindian, $64.25 \%$ for Asian, 0.0\% for Austronesian, 64.12\% for Black, 85.29\% for Caucasoid, $71.07 \%$ for Hispanic, $72.82 \%$ for Mestizo and $70.45 \%$ for Polynesian. In addition, such epitopes presented population coverages higher than 50\% for different continents and subcontinents of the world: North America, South America, Europe, West Africa, Central Africa, North Africa, South Africa, Northeast Asia, South Asia, Eats Asia, Southeast Asia, Southwest Asia and Oceania. Relevantly, selected epitopes contained in the consensus ZIKV NS5 presented population coverages of $81.76 \%, 68.5 \%, 64.63 \%, 65.32 \%$ and $59.37 \%$ for Europe, North America, South America, East Asia and Southeast Asia, respectively (Table 3). These results indicate that the consensus NS5 protein proposed in this study is a promising antigen with regard to induce cellular immune response against ZIKV and DENV in different human populations of the world.

\section{D NS5 model and epitope distribution on the protein}

The structure computed for the ZIKV NS5 consensus was shown to be similar to a previous reported ZIKV NS5 structure deposited in PDB library [22]. As shown in Table 4, a high level of identity and a high TM-score were found between the consensus protein and the top ranked similar, a previous reported ZIKV NS5 protein. In addition, those 19 epitopes found to be $100 \%$ conserved among ZIKV lineages and DENV serotypes were shown to be homogeneously distributed along the protein structure (Fig. 3). Some overlapping epitopes were found in the protein and thus, their distribution is shown in regions. Three regions containing epitopes specific for HLA-A presentation were found (Fig. 3a). One of them is located at the MTase domain and contains only one epitope (Region 1, epitope LSRNSTHEMY) (Fig. 3b). Two other regions (2 and 3) which contain together six epitopes are located at the RdRp domain (Fig. 3c). Two epitopes are located in region 2 (Region 2, epitopes CVYNMMGKR and CVYNMMGKREK) and four in region 3 (Region 3, epitopes AIWYMWLGAR, WYMWLGAR, RAIWYMWL GAR and IWYMWLGAR). One region containing only one epitope specific for HLA-B presentation is located at the RdRp domain (Region 1, epitope LEFEALGF) (Fig. 3d). Five regions containing epitopes specific for HLA-C presentation were found (Fig. 3e). Two of them are located at the MTase domain. Region 1 contains three epitopes (Region 1, epitopes SRNSTHEM, SRNSTHEMY and SRNSTHEMYW) and region 2 contains one epitope (Region 2, epitope GRGGWSYY) (Fig. 3f). Three other regions are located at the $\mathrm{RdRp}$ domain: region 3 contains 
Table 2 ZIKV NS5 predicted epitopes 100\% conserved among ZIKV lineages and DENV serotypes and their respective population coverage rates (\%) considering the Brazilian population

\begin{tabular}{|c|c|c|c|}
\hline Epitope & Location $^{a}$ & Alleles & Population coverage \\
\hline LSRNSTHEMY & $211-220$ & HLA-A*01:01 & $13.87 \%$ \\
\hline CVYNMMGKR & $451-459$ & HLA-A*03:01, HLA-A*31:01, HLA-A*33:03 & $12.06 \%$ \\
\hline CVYNMMGKREK & $451-461$ & HLA-A*03:01, HLA-A*11:01 & $12.06 \%$ \\
\hline AIWYMWLGAR & $474-483$ & HLA-A*31:01 & $11.06 \%$ \\
\hline WYMWLGAR & $476-483$ & HLA-A*31:01, HLA-A*33:03, HLA-C*04:01 & $33.73 \%$ \\
\hline RAIWYMWLGAR & $473-483$ & HLA-A*33:03 & $3.9 \%$ \\
\hline IWYMWLGAR & $475-483$ & HLA-A*31:01, HLA-A*33:03 & $11.06 \%$ \\
\hline LEFEALGF & $485-492$ & HLA-B*18:01, HLA-C*04:01 & $8.0 \%$ \\
\hline YADDTAGW & $532-539$ & HLA-C*03:03, HLA-C*05:01, HLA-C*08:02, HLA-C*17:01 & $9.64 \%$ \\
\hline RAIWYMWL & $473-480$ & HLA-C*03:03, HLA-C*07:02, HLA-C*15:02 & $9.64 \%$ \\
\hline YADDTAGWDT & $532-541$ & HLA-C*05:01, HLA-C*08:02 & $9.7 \%$ \\
\hline YADDTAGWDTR & $532-542$ & HLA-C*05:01, HLA-C*08:02 & $9.7 \%$ \\
\hline YADDTAGWD & $532-540$ & HLA-C*05:01, HLA-C*12:03 & $9.7 \%$ \\
\hline ADDTAGWD & $533-540$ & HLA-C*05:01 & $9.7 \%$ \\
\hline GRGGWSYY & $83-90$ & HLA-C*07:01, HLA-C*07:02 & $23.83 \%$ \\
\hline SRNSTHEM & $212-219$ & HLA-C*07:01, HLA-C*07:02 & $23.83 \%$ \\
\hline SRNSTHEMY & $212-220$ & HLA-C*07:01, HLA-C*07:02 & $23.83 \%$ \\
\hline SRNSTHEMYW & $212-221$ & HLA-C*07:01 & $23.83 \%$ \\
\hline SRAIWYMWL & $472-480$ & HLA-C*07:02 & $19.15 \%$ \\
\hline
\end{tabular}

a Location of the predicted epitope into the ZIKV NS5 consensus amino acid sequence

three epitopes (Region 3, epitopes RAIWYMWL, WYMWLGAR and SRAIWYMWL), region 4 contains one epitope (Region 4, epitope LEFEALGF) and region 5 contains four epitopes (Region 5, epitopes YADDTAGW DT, YADDTAGWDTR, YADDTAGWD and ADDTAGW D) (Fig. 3g).

\section{Discussion}

Zika virus (ZIKV) is a new emergent pathogen previously associated with mild infections which took off to cause microcephaly in babies and Guillain-Barré syndrome in the Americas [3-5]. Dengue virus (DENV1-4) [2] infects around 390 million of people per year. These two Flavivirus consists a world public health concern for which there is not specific treatment. There is not a vaccine approved for controlling ZIKV in humans. The only anti-DENV vaccine candidate approved for use in humans is based on a chimera between DENV and Yellow fever virus (YFV), in which envelope proteins are the only DENV-specific antigens [6-10]. Such proteins are main targets for humoral immune response. However, especially for DENV2, the vaccine formulation based on those chimeric viruses did not achieve the expected protective efficacy in phase III clinical trials. In addition, it was reported to present higher incidence of hospitalization among children [10]. Such increased hospitalization may be related to antibody dependent enhancement. Importantly, enhancement of ZIKV infection mediated by DENV-specific antibodies was observed in vitro [11-14]. Thus, the risk of inducing antibody-mediated enhancement of infection between these two major arboviruses will depend on the profile of immune response induced.

It was recently shown that protection against DENV is achieved independently of humoral immunity [19]. CD4 ${ }^{+}$and $\mathrm{CD}^{+} \mathrm{T}$ lymphocytes targeting non-structural protein 5 (NS5) were shown to be essential for protection. When a recombinant purified form of NS5 protein was used as a vaccine antigen, $70 \%$ of protection was achieved on a mouse model [20]. In this study, we aimed to design a vaccine antigen capable of inducing crossprotective cellular immunity against DENV and ZIKV. As the NS5 protein is the most conserved antigen between DENV and ZIKV, a ZIKV consensus amino acid sequence was shown to present a relevant number of epitopes $100 \%$ conserved among DENV serotypes and ZIKV lineages to be presented by HLA (human leucocyte antigen) alleles of different populations of the world. The designed antigen was shown to present a homogeneous distribution of epitopes and its predicted 3D model was shown to fit with the structure of a ZIKV native NS5.

A comprehensive phylogenetic analysis based on NS5 protein amino acid sequences from ZIKV and DENV isolates from different continents was carried out. It was 


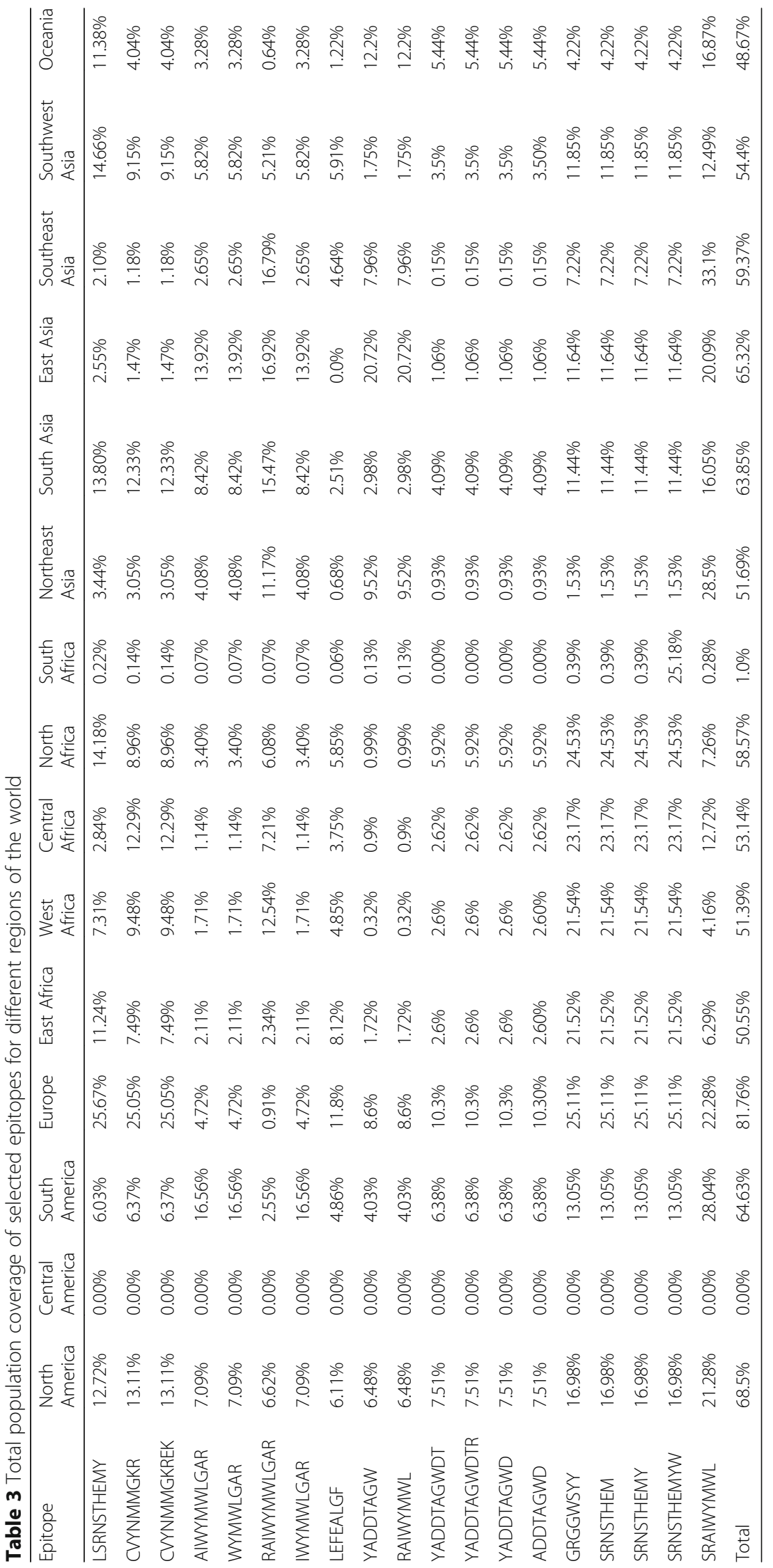


Table 4 Molecular modeling results of the amino acid consensus sequence of ZIKV NS5

\begin{tabular}{|c|c|c|c|c|c|}
\hline $\operatorname{Rank}^{a}$ & PDB Hit ${ }^{b}$ & TM-score ${ }^{c}$ & $\mathrm{RM} \mathrm{SD}^{\mathrm{d}}$ & IDEN $^{e}$ & $\operatorname{Cov}^{f}$ \\
\hline 1 & $5 \mathrm{u} 0 \mathrm{bA}$ & 0.977 & 0.30 & 0.960 & 0.978 \\
\hline 2 & $4 \mathrm{k} 6 \mathrm{~mA}$ & 0.972 & 0.93 & 0.694 & 0.980 \\
\hline 3 & $4 v 0 q A$ & 0.714 & 4.18 & 0.518 & 0.784 \\
\hline 4 & $2 \mathrm{hfzA}$ & 0.616 & 2.98 & 0.689 & 0.660 \\
\hline 5 & $2 j 7 u A$ & 0.615 & 1.87 & 0.677 & 0.631 \\
\hline 6 & $1 \mathrm{~s} 4 \mathrm{fA}$ & 0.517 & 3.62 & 0.139 & 0.568 \\
\hline 7 & $2 c j q A$ & 0.516 & 3.57 & 0.151 & 0.570 \\
\hline 8 & $2 \mathrm{hcs} A$ & 0.512 & 2.41 & 0.694 & 0.534 \\
\hline 9 & $1 y v x A$ & 0.501 & 4.16 & 0.148 & 0.566 \\
\hline 10 & $3 c j 0 B$ & 0.497 & 4.24 & 0.132 & 0.564 \\
\hline
\end{tabular}

ananking of proteins is based on TM-score of the structural alignment between the query structure and known structures in the PDB library

${ }^{\text {b}}$ Digital object identifier of the top 10 proteins deposited in the PDB library which have the closest structural similarity to the ZIKV conosensus NS5;

'Alignment score with the most similar protein structure found in PDB library; ${ }^{\mathrm{d}}$ Root-mean-square deviation of atomic positions (root-mean-square deviation, RMSD) between residues that are structurally aligned by TM-align ePercentage of sequence identity in the structurally aligned region; ${ }^{\text {f }} \mathrm{Cov}$ represents the coverage of the alignment by TM-align and is equal to the number of structurally aligned residues divided by length of the query protein shown that sequences from American ZIKV isolates grouped with those from isolates of the ZIKV Asian lineage. This corroborates the recent literature [23]. In addition, DENV and ZIKV NS5 proteins were shown to have diverged before their separation into different viral species and evolutionary distances could not be inferred based on phylogenetic tree. However, the consensus ZIKV NS5 protein was shown to share different numbers of epitopes with each of your homologous proteins. This result indicates that the proposed antigen could induce crossreactive immune responses with different levels of intensity to ZIKV, DEN1, DENV2, DENV3 and DENV4.

The consensus NS5 protein was designed based on ZIKV isolates from throughout the world. Attempts to produce a ZIKV/DENV consensus resulted in a nonstable protein model (data not shown). Nevertheless, nineteen epitopes specific for presentation by class I HLA were found to be $100 \%$ conserved among DENV serotypes and ZIKV lineages. Population coverages of such epitopes with regard to different human populations in the world were shown to be relevant. Predicted vaccine coverage for populations from several tropical areas were higher than $50 \%$. This is an important characteristic of the designed antigen considering that HLA restriction is a major challenge in vaccine development, which may significantly

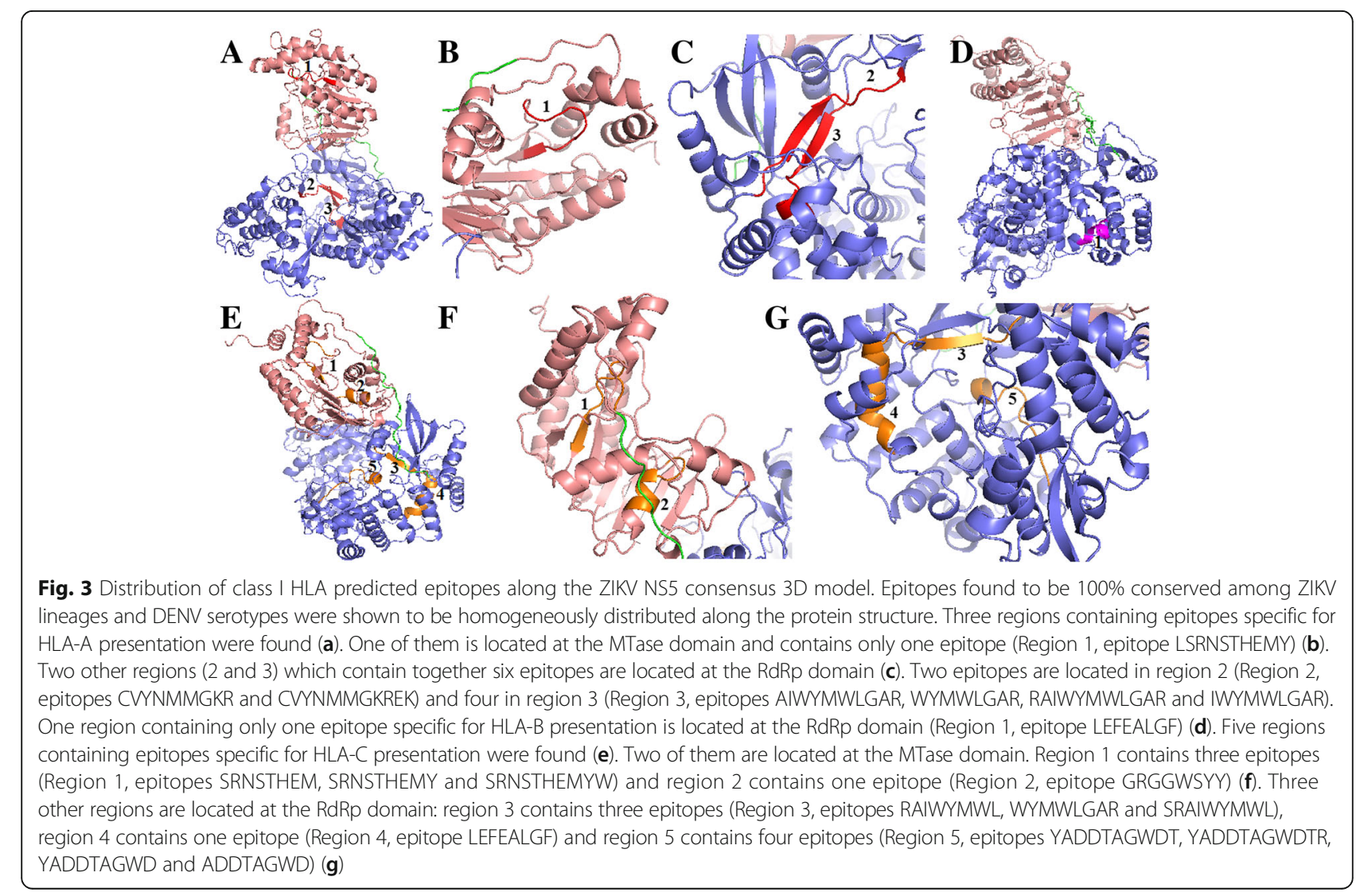


affect vaccine efficacy and effectiveness among human populations of different regions.

The 100\% conserved epitopes were shown to be homogeneously distributed along protein domains. Although such amino acid sequences may be used in a polyepitope development project, their homogeneous distribution along the protein structure is important for using the whole antigen. As NS5 protein is a major target for $\mathrm{CD} 4^{+}$ and $\mathrm{CD}^{+} \mathrm{T}$ lymphocytes, using the whole antigen such as we previously reported [19] would be a promising strategy in order to induce a significant immune response against the conserved epitopes. Thus, we conclude that the proposed antigen has the potential to induce protective cellular immune response to ZIKV and DENV in different human populations in the world.

\section{Conclusions}

In the end of the analysis we conclude that NS5 consensus protein have the potential to figurate a vaccine antigen to induce cross protection to ZIKV end DENV in different human populations of the world. The next step in this process is to analyze real immune response for this antigen in mouse model and see how the immune response process will proceed.

\section{Additional file}

Additional file 1: Table S1. -ZIKV Sequences. The sequences are named with virus species, accession number and country of isolation. Table S2 DENV1 sequences. The sequences are named with virus species and serotype, accession number and country of isolation. Table S3 - DENV2 sequences. The sequences are named with virus species and serotype, accession number and country of isolation. Table S4 - DENV3 sequences. The sequences are named with virus species and serotype, accession number and country of isolation. Table S5 - DENV4 sequences. The sequences are named with virus species and serotype, accession number and country of isolation (DOCX $18 \mathrm{~kb}$ )

\section{Acknowledgements}

We appreciate the financial support of the International Society for Infectious Diseases. We are also thankful to CNPq by providing undergraduate research fellowship for Lorrany dos Santos Franco.

\section{Ethical approval and consent to participate}

Not applicable. We did not carry out experiments that need ethical approval or consent to participate.

\section{Funding}

This research work was funded by International Society of Infectious Diseases.

\section{Availability of data and materials}

We are submitting supporting data together with this manuscript.

\section{Author's contributions}

LSF carried out analyses and interpreted the data generated; POV and JHA conceived the study and interpreted the data generated. All authors wrote the manuscript. All authors read and approved the final version of the manuscript.

Consent for publication

All the authors of this manuscript agreed to publish this research work.
Competing interests

All authors declare that they have no competing interests.

\section{Publisher's Note}

Springer Nature remains neutral with regard to jurisdictional claims in published maps and institutional affiliations.

Received: 9 August 2017 Accepted: 17 November 2017

Published online: 23 November 2017

\section{References}

1. Fields Virology, 5th Edition. D. M. Knipe and P. M. Howley, Eds. Philadelphia: Lippincott-Raven Publishers; 2007

2. Bhatt $\mathrm{S}$, Gething PW, Brady OJ, Messina JP, Farlow AW, Moyes $C L$, et al. The global distribution and burden of dengue. Nature. 2013;496:504-7.

3. Calvet G, Aguiar RS, Melo ASO, Sampaio SA, de Filippis I, Fabri A, et al. Detection and sequencing of Zika virus from amniotic fluid of fetuses with microcephaly in Brazil: a case study. Lancet Infect. Dis. [Internet]. 2016 [cited 2016 Feb 20]; Available from: http://www.ncbi.n/m.nih.gov/pubmed/26897108.

4. Wikan N, Smith DR. Zika virus: history of a newly emerging arbovirus. Lancet Infect. Dis. [Internet]. 2016 [cited 2017 Apr 12];16:e119-e126. Available from: http://www.ncbi.nlm.nih.gov/pubmed/27282424

5. Areas with Zika | Zika virus | CDC [Internet]. [cited 2016 Mar 15]. Available from: http://www.cdc.gov/zika/geo/

6. Guirakhoo F, Arroyo J, Pugachev K V, Miller C, Zhang ZX, Weltzin R, et al. Construction, safety, and immunogenicity in nonhuman primates of a chimeric yellow fever-dengue virus tetravalent vaccine. J. Virol. [Internet]. 2001 [cited 2014 Nov 24];75:7290-7304. Available from: http://www.pubmedcentral.nih. gov/articlerender.fcgi?artid=114964\&tool=pmcentrez\&rendertype=abstract

7. Guirakhoo F, Weltzin R, Chambers TJ, Zhang ZX, Soike K, Ratterree M, et al. Recombinant chimeric yellow fever-dengue type 2 virus is immunogenic and protective in nonhuman primates. J. Virol. [Internet]. 2000 [cited 2015 Aug 7];74:5477-5485. Available from: http://www.pubmedcentral.nih.gov/ articlerender.fcgi?artid $=112032 \&$ tool=pmcentrez\&rendertype $=$ abstract

8. Guirakhoo F, Pugachev K, Zhang Z, Myers G, Levenbook I, Draper K, et al. Safety and efficacy of chimeric yellow Fever-dengue virus tetravalent vaccine formulations in nonhuman primates. J. Virol. [Internet]. 2004 [cited 2015 Aug 7];78:4761-4775. Available from: http://www.pubmedcentral.nih. gov/articlerender.fcgi?artid=387722\&tool=pmcentrez\&rendertype=abstract

9. Villar L, Dayan GH, Arredondo-García JL, Rivera DM, Cunha R, Deseda C, et al. Efficacy of a Tetravalent Dengue Vaccine in Children in Latin America. N. Engl. J. Med. [Internet]. 2014 [cited 2014 Nov 4];372:141103114505002. Available from: http://www.ncbi.n/m.nih.gov/pubmed/25365753.

10. Hadinegoro SR, Arredondo-García JL, Capeding MR, Deseda C, Chotpitayasunondh T, Dietze R, et al. Efficacy and Long-Term Safety of a Dengue Vaccine in Regions of Endemic Disease. N. Engl. J. Med. [Internet]. 2015 [cited 2017 may 23];373:1195-1206. Available from: http://www.ncbi. nlm.nih.gov/pubmed/26214039.

11. Paul LM, Carlin ER, Jenkins MM, Tan AL, Barcellona CM, Nicholson CO, et al. Dengue virus antibodies enhance Zika virus infection. Clin. Transl. Immunol. [Internet]. 2016 [cited 2017 Feb 19];5:e117. Available from: http://www.ncbi. nlm.nih.gov/pubmed/28090318.

12. Priyamvada L, Quicke KM, Hudson WH, Onlamoon N, Sewatanon J, Edupuganti $\mathrm{S}$, et al. Human antibody responses after dengue virus infection are highly cross-reactive to Zika virus. Proc. Natl. Acad. Sci. [Internet]. National Academy of Sciences; 2016 [cited 2016 Aug 8];113:7852-7857. Available from: http://www.pnas.org/lookup/doi/10.1073/pnas.1607931113

13. Castanha PM, Nascimento EJM, Cynthia B, Cordeiro MT, de Carvalho O V., de Mendonça LR, et al. Dengue virus (DENV)-specific antibodies enhance Brazilian Zika virus (ZIKV) infection. J. Infect. Dis. [Internet]. 2016 [cited 2017 Feb 19];ijw638. Available from: http://www.ncbi.nlm.nih.gov/pubmed/28039355.

14. Dejnirattisai W, Supasa P, Wongwiwat W, Rouvinski A, Barba-Spaeth G, Duangchinda $T$, et al. Dengue virus sero-cross-reactivity drives antibodydependent enhancement of infection with zika virus. Nat. Immunol. [Internet]. Nature Research; 2016 [cited 2016 Aug 8]; Available from: http://www.nature. com/doifinder/10.1038/ni.3515

15. Gil L, López C, Blanco A, Lazo L, Martín J, Valdés I, et al. The cellular immune response plays an important role in protecting against dengue virus in the mouse encephalitis model. Viral Immunol. [Internet]. 2009 [cited 2015 Apr 16];22:23-30. Available from: http://www.ncbi.nlm.nih.gov/pubmed/19210225. 
16. Rivino L, Kumaran EAP, Jovanovic V, Nadua K, Teo EW, Pang SW, et al. Differential targeting of viral components by CD4+ versus CD8+ Tlymphocytes in dengue virus infection. J. Virol. [nternet]. 2013 [cited 2015 Feb 13];87:2693-2706. Available from: http://www.pubmedcentral.nih.gov/articlerender. fcgi?artid $=3571409 \&$ tool $=$ pmcentrez\&rendertype $=$ abstract

17. Yoshida T, Omatsu T, Saito A, Katakai Y, Iwasaki Y, Kurosawa T, et al. Dynamics of cellular immune responses in the acute phase of dengue virus infection. Arch. Virol. [Internet]. 2013 [cited 2015 Apr 16];158:1209-1220. Available from: http://www.ncbi.nlm.nih.gov/pubmed/23381396.

18. Weiskopf D, Angelo MA, Bangs DJ, Sidney J, Paul S, Peters B, et al. The human CD8+ T cell responses induced by a live attenuated tetravalent dengue vaccine are directed against highly conserved epitopes. J. Virol. [Internet]. 2014 [cited 2014 Nov 12]; Available from: http:/www.ncbi.nlm. nih.gov/pubmed/25320311.

19. Amorim JH, Dos Santos Alves RP, Bizerra R, Araújo Pereira S, Ramos Pereira $\mathrm{L}$, Nascimento Fabris $\mathrm{DL}$, et al. antibodies are not required to a protective immune response against dengue virus elicited in a mouse encephalitis model. Virology. 2016;487:41-9.

20. Alves RPDS, Pereira L, Fabris DLN, Salvador FS, Santos RA, Zanotto PM de A, et al. Production of a recombinant Dengue virus 2 NS5 protein and the potential use as a vaccine antigen. Clin. Vaccine Immunol. [Internet]. 2016 [cited 2016 Apr 22]; Available from: http://cvi.asm.org/content/early/2016/ 03/28/CVI.00081-16.abstract

21. Tamura K, Peterson D, Peterson N, Stecher G, Nei M, Kumar S. MEGA5: Molecular Evolutionary Genetics Analysis Using Maximum Likelihood, Evolutionary Distance, and Maximum Parsimony Methods. Mol Biol Evol. 2011;28:2731-9.

22. Zhao B, Yi G, Du F, Chuang Y-C, Vaughan RC, Sankaran B, et al. ARTICLE Structure and function of the Zika virus full-length NS5 protein. Nat. Commun. [Internet]. 2017 [cited 2017 Nov 3];8. Available from: https:/www.nature.com/ articles/ncomms 14762.pdf

23. Basarab M, Bowman C, Aarons EJ, Cropley I. Vírus da Zika. BMJ. 2016:1-6.

\section{Submit your next manuscript to BioMed Central and we will help you at every step:}

- We accept pre-submission inquiries

- Our selector tool helps you to find the most relevant journal

- We provide round the clock customer support

- Convenient online submission

- Thorough peer review

- Inclusion in PubMed and all major indexing services

- Maximum visibility for your research

Submit your manuscript at www.biomedcentral.com/submit

) Biomed Central 